Research Article

\title{
A comparative study between Poly (lactide-co-glycolide) derived composite sintered microsphere scaffolds for bone tissue engineering
}

\author{
Shalumon K.T. ${ }^{\text {**}}$, Jyh-Ping Chen ${ }^{\mathrm{a}, \mathrm{b}}$, Chialin sheu ${ }^{\mathrm{a}}$ \\ ${ }^{a}$ Department of Chemical and Materials Engineering, Chang Gung University, Kweishan, Taoyuan, \\ Taiwan 333, ROC \\ ${ }^{\mathrm{b}}$ Research Center for Industry of Human Ecology, Chang Gung University of Science and \\ Technology, Kweishan, Taoyuan 333, Taiwan, ROC
}

*Corresponding author Tel: +886-3-2118800; fax: +886-3-2118668; E-mail: shalumon@gmail.com, shalumon@mail.cgu.edu.tw

Received: 8 April 2014 / Published: 6 June 2014

\begin{abstract}
Microsphere based scaffolds can be suggested as alternative to nanofibrous based scaffolds, owing to high cell infiltration and mechanical stability. Poly (lactide-co-glycolide) (PLGA) with lactide to glycolide ratio 85:15 was used to make virgin and composite microsphere based scaffolds. Nano Hydroxyapatite (nHAP) was synthesized, characterized and in-situ incorporated into microspheres to make $10 \%$ nHAP composite scaffolds. $250-500 \mu \mathrm{m}$ microspheres were used for making virgin, composite and a combination of virgin/composite (osteochondral) scaffolds in disc shape. Morphology of the scaffolds was determined using microscopy whereas porosity was evaluated using specific gravity method and pore size analyzer. nHAP was characterized using Transmission electron Microscopy (TEM) and presence of nHAP in composite scaffolds was examined using Fourier Transform Infrared Spectroscopy (FTIR), X-ray diffraction spectroscopy (XRD) and Energy Dispersive Spectrum (EDS). Effect of nHAP in physical properties of scaffolds was investigated through Thermo gravimetrical Analysis (TGA). Biocompatibility and cell infiltration of the scaffolds was evaluated using culturing with rabbit bone marrow stem cells (BMSC) followed by microscopic evaluation. A meaningful comparison between three scaffold types will provide a conclusive declaration for the selection of ideal scaffold for bone tissue engineering.
\end{abstract}

Keywords: Microsphere; Composite scaffolds; Porosity; PLGA; Hydroxyapatite 


\section{Introduction}

Tissue engineering using nano and micro-scale biomaterials is a promising approach for the regeneration of damaged tissues and organs. In bone tissue engineering, scaffolds should provide a temporary environment for bone-forming cells to adhere, penetrate and hence to form new bone tissues. Nanoscale materials are good candidate for cell proliferation but poor in mechanical stability and cell infiltration. Due to the minimum pore size of nanofibrous scaffolds, its potential in the fabrication of scaffolds is bit questionable.

Tissue engineering involves the right mix of cells and suitably shaped biocompatible scaffolds with osteoconductive/inductive factors (Horch et al., 2012). Lack of suitable-well designed scaffolds, which allows the three dimensional growth of cells in the interiors is one of the major challenges in tissue engineering (Temenoff \& Mikos, 2000). Microsphere based scaffolds can facilitate the mechanical integrity and porosity required for an ideal scaffold. Pore interconnectivity could also help to promote the vascularization process and hence fulfil the angiogenesis completely. Composite polymers can give added beneficial outcome for specific organ development such as bone, by incorporating ceramic materials such as hydroxyapatite and tri calcium phosphate. This proposed plan of work deals with the right mix of osteoconductive ceramic particles (nHAP) in PLGA microspheres, while maintaining the porosity and mechanical integrity unchanged. The effect of nHAP in composite scaffold was compared with virgin scaffold and the interface characteristics of virgin and composite scaffolds for osteochondral development was examined by developing a virgin/composite combined scaffold. The porosity of the scaffolds was evaluated in terms of cell accessibility into the interiors of the scaffold, using BMSC. More detailed studies are needed to assess the optimum porosity and concentration of nHAP in scaffolds, to develop an ideal bone tissue engineering scaffold.

\section{Results and Discussions}

\subsection{Microsphere scaffolds}

Figure 1 (a-d) represents microscopic images of virgin microspheres and (e-h) denotes the same size range for composites. a\&e, b\&f, c\&g and d\&h respectively represents microspheres in the size 88-250, $251-500,501-750$ and $>750 \mu \mathrm{m}$. Spheres in the range $250-500 \mu \mathrm{m}$ was selected for the fabrication of disc shaped scaffolds. Fig.1 (i,j\&K) and $(1, m \& n)$ respectively represents the SEM and photographic images of virgin, composite and interface (osteochondral) scaffolds. The motive behind the development of osteochondral scaffold is to identify the interface properties of a non osteo-conductive and osteo-conductive material, towards tissue regeneration. The porous-interlinked morphology of scaffolds explains the potential of such scaffolds in the repair of osteochondral defects. 
Figure 1. (a-h) represents various size ranges of microspheres of virgin (a-d) and composite (e-h) spheres. (i\&l), (j\&m) and (k\&n) respectively represents the microscopic and photographic images of virgin, composite and osteochondral scaffolds.
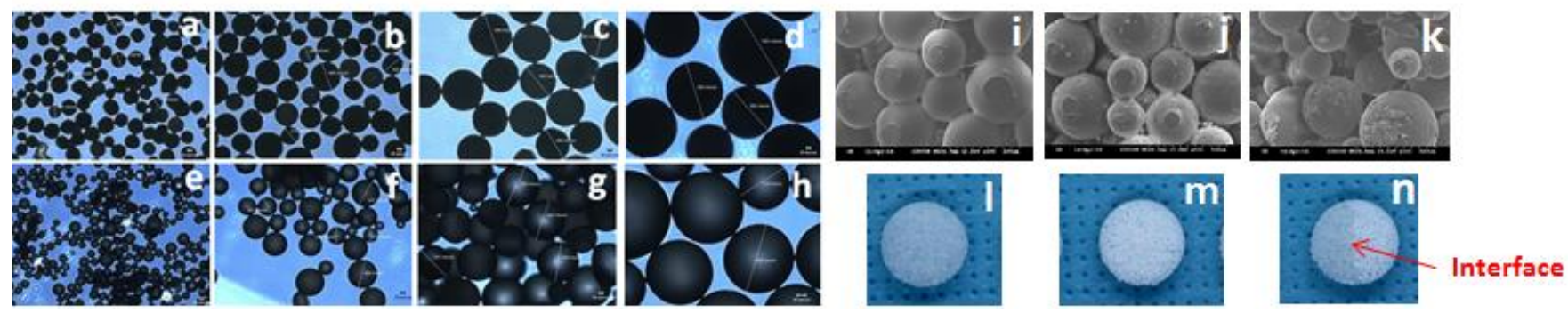

\subsection{Characterization of scaffolds}

\subsubsection{Microscopic, spectroscopic and thermal characterization}

nHAP was characterized using TEM. An average particle size of $15 \mathrm{~nm}$ was observed as diameter of the particle (Fig.2a). In-situ incorporation of nHAP into PLGA microspheres was further confirmed through FTIR and XRD(Shalumon et al., 2010). The peaks at broad range of 3500 and $633 \mathrm{~cm}^{-1}$ correspond to the hydroxyl vibrations whereas strong and weak phosphate stretching vibrations were observed at 1030, 1081, 968 and $602 \mathrm{~cm}^{-1}$ (Fig.2b). Crystallinity of nHAP was confirmed by the presence of prominent peaks at $2 \Theta$ value 26 (002 plane) and 32 (211 plane)(Chen, Tsai, \& Liao, 2013). Composite and osteochondral scaffolds were further confirmed by the presence of nHAP peaks and compared with JCPDC 090432 (Fig.2c). Quantitative estimation of nHAP in composite scaffolds was evaluated through comparative thermal decomposition of virgin and composite scaffolds (Fig.2d). Virgin scaffold decomposed completely at $\sim 480^{\circ} \mathrm{C}$ whereas composite scaffold retained $9.6 \%$ of the initial mass, depicting the actual concentration of nHAP in it. Apart from XRD and FTIR, nHAP content in composite and osteochondral scaffolds were cross confirmed with EDS analysis. Both the scaffolds show a $\mathrm{Ca} / \mathrm{P}$ stoichiometric ratio of 1.652 (e) and 1.631 (f) respectively for composite and osteochondral scaffolds, in close relation with the ideal value 1.67.

Figure 2. (a) is TEM image of nHAP and (b\&c) represents FTIR and XRD patterns of virgin, composite and osteochondral scaffolds. (d) is the thermal decomposition curve of virgin and composite scaffolds, to estimate the actual concentration of nHAP in composite scaffold. (e\&f) are the EDS spectrum of virgin and composite scaffold to confirm the stoichiometric $\mathrm{Ca} / \mathrm{P}$ ratio of prepared nHAP. 

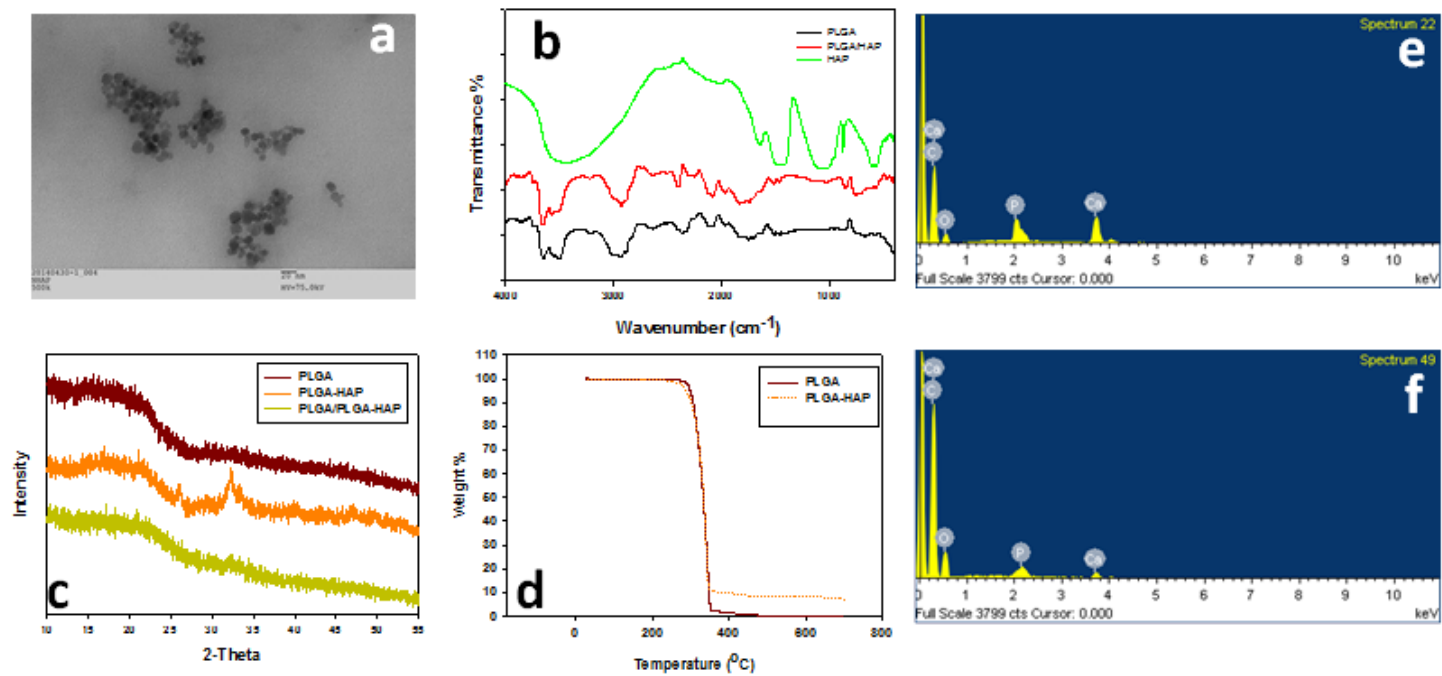

\subsubsection{Porosity of scaffolds}

Specific gravity method was used for the determination of porosity of scaffolds. Dry weight, wet weight and volume of the scaffold were used to determine the porosity percentage of each scaffold. Virgin, composite and osteochondral scaffolds shows average porosity of 153, 137 and 136\% respectively whereas pore size distribution showed mean pore diameter $296.5,263.9$ and $282.8 \mu \mathrm{m}$. The higher porosity is due to more percentage of void spaces between each sphere. Each sphere should ideally connect with several other spheres and the spherical nature creates maximum empty space suitable enough for cell penetration. The average pore size, which is greater than $100 \mu \mathrm{m}$, facilitates easier cell access into the interiors of the scaffold.

\subsection{Cell infiltration evaluation}

The success of biomaterials solely depends on the biocompatibility, degradability and porosity of the material. PLGA is a well-known biodegradable/biocompatible material, but the morphology of the scaffold determines the regeneration ability of scaffold made up of PLGA. Here rabbit BMSC were seeded onto the scaffolds for 24 and 72 hours, to assess the cell-material interaction and cell infiltration. $\mathrm{a}, \mathrm{b}, \mathrm{c}$ and $\mathrm{a} 1, \mathrm{~b} 1, \mathrm{c} 1$ respectively represents surface and cross sectional view of BMSCs on the interiors of (a) virgin (b) composite and (c) osteochondral scaffolds. a2, b2 and c2 are the z-stacked cell infiltration images by confocal microscope at 3 days of cell infiltration. Arrows indicates the cell filopdial extensions on the sphere surface. It is evident that pores in the scaffolds are larger enough for cell access and must be suitable enough for tissue regeneration using stem cells. More experiments are required to completely understand the regeneration potential of scaffolds. 
Figure 3. a, b, c and a1, b1, c1 respectively represents surface and cross sectional view of BMSCs on the interiors of (a) virgin (b) composite and (c) osteochondral scaffolds. a2, b2 and c2 is the z-stacked cell infiltration images by confocal microscope. Arrows indicates the filopdial extensions of cells on the sphere surface.

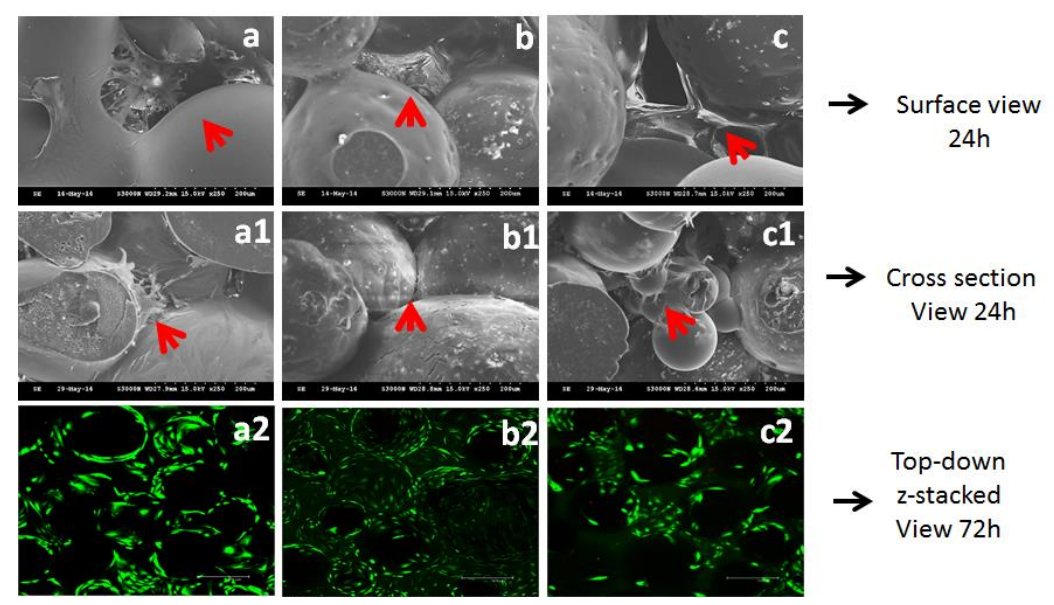

\section{Experimental Section}

\subsection{Materials}

All the chemicals were used as purchased without further purification.

\subsection{Preparation of microsphere scaffolds}

PLGA microspheres with and without nHAP was prepared by emulsion method (Yang, Chia, \& Chung, 2000). Briefly, 14\% PLGA solution was prepared in methylene chloride (DCM) followed by slow pouring into 1 litre polyvinyl alcohol (PVA) solution (0.5\%) stirring at 360 RPM and continued overnight. The microspheres formed in the solution were washed, dried and sorted using metalic sieves. For making composite spheres, 10\% nHAP (W/W) was incorporated into PLGA solution before pouring into PVA solution. Selected spheres of size $250-500 \mu \mathrm{m}$ was heat sintered in stainless steel mould at $85^{\circ} \mathrm{C}$ ( 2 hours) to produce three types of scaffolds. The first and second scaffold is made up of PLGA (virgin) and PLGA with 10\% nHAP (composite) microspheres whereas third one is the interface combination of both (osteochondral).

\subsection{Characterization of scaffolds}

The particle size of prepared nHAP was measured using TEM (Hitachi, Japan). Size, shape and morphology of the microsphere scaffolds were observed through light microscope and SEM (Hitachi, Japan) The FTIR spectrum of virgin microspheres was compared with nHAP incorporated counterparts using a Horiba FT-730 spectrometer (Horiba, Ltd., Kyoto, Japan). 
3.4 Evaluation of nHAP content in scaffolds.

XRD spectrum of virgin, composite and osteochondral scaffolds was recorded using a Siemens D5005 Xray diffractometer (Bruker AXS, Karlsruhe, Germany) at a speed of $2 \%$ min and with a $2 \Theta$ range 5-35 . Presence of $\mathrm{nHAP}$ in composite scaffolds was cross verified by atomic percentage calculation of energy dispersive analysis (EDX) (HORIBA EX-250, Japan). Quantitative measurement of nHAP in composite scaffolds was performed by TGA. Scaffolds were heated from room temperature to $700^{\circ} \mathrm{C}$ with a heating rate of $10^{\circ} \mathrm{C} / \mathrm{min}$.

\subsection{Porosity of scaffolds}

Porosity of the scaffolds was measured using modified specific gravity method (Kathuria, Tripathi, Kar, \& Kumar, 2009) of Archimedes principle. Cylindrical scaffolds were immersed in 100\% ethanol prior to wet-dry weight measurements. Porosity of the scaffolds were measured using the equation

$$
\operatorname{Porosity}(\%)=\frac{W w-W d / D}{\text { Vscaffold }} \times 100
$$

Where Ww, Wd, D and Vscaffold are wet weight of the scaffold, dry weight of the scaffold, density of ethanol and volume of scaffold respectively. Mean pore diameter of the scaffolds was measured using pore size analyzer (PMI, USA).

3.6 Cell infiltration studies using bone marrow stem cells

Rabbit BMSCs were cultured on cylindrical scaffolds for 24 hours at a seeding rate of $1 \times 10^{5}$ cells/ scaffold. Morphology of the cells on scaffold surface and interiors was analyzed using surface/cross section SEM and Z-stacking by confocal microscope.

\section{Conclusions}

PLGA based microsphere scaffolds were prepared by emulsion method and scaffolds were fabricated by heat sintering. Three types of scaffolds, virgin, composite and osteochondral composite scaffolds were prepared and characterized. BMSC were seeded into the scaffold interiors to evaluate infiltration potential and regeneration capability. Even at early stages of cell seeding, cells are firmly attaching and infiltrating to the microsphere interiors. Further studies on osteochondral scaffolds will be helpful to understand the selective regeneration scenario on it. Introduction of inductive/conductive factors on separate compartments of the osteochondral scaffold will be a viable method for regeneration process. Such scaffolds are expected to be better candidates for bone related tissue engineering applications. 


\section{Acknowledgments}

We are thankful for the financial supports from National Science Council, ROC (NSC-100-2221-E182-024 and NSC-101-2221-E-182-029) and Chang Gung Memorial Hospital (CMRPD1B0241 and CMRPD1B0242).

\section{Conflicts of Interest}

The authors declare no conflict of interest

\section{References and Notes}

1. Chen, J.-P., Tsai, M.-J., \& Liao, H.-T. (2013). Incorporation of biphasic calcium phosphate microparticles in injectable thermoresponsive hydrogel modulates bone cell proliferation and differentiation. Colloids and Surfaces B: Biointerfaces, 110(0), 120-129. doi: http://dx.doi.org/10.1016/j.colsurfb.2013.04.028

2. Horch, R. E., Kneser, U., Polykandriotis, E., Schmidt, V. J., Sun, J., \& Arkudas, A. (2012). Tissue engineering and regenerative medicine -where do we stand? J Cell Mol Med, 16(6), 1157-1165. doi: $10.1111 / \mathrm{j} .1582-4934.2012 .01564 . \mathrm{x}$

3. Kathuria, N., Tripathi, A., Kar, K. K., \& Kumar, A. (2009). Synthesis and characterization of elastic and macroporous chitosan-gelatin cryogels for tissue engineering. Acta Biomater, 5(1), 406-418. doi: http://dx.doi.org/10.1016/j.actbio.2008.07.009

4. Shalumon, K. T., Binulal, N. S., Deepthy, M., Jayakumar, R., Manzoor, K., \& Nair, S. V. (2010). Preparation, Characterization and Cell Attachment Studies of Electrospun Multi-scale Poly(caprolactone) Fibrous Scaffolds for Tissue Engineering. Journal of Macromolecular Science, Part A, 48(1), 21-30. doi: 10.1080/10601325.2011.528288

5. Temenoff, J. S., \& Mikos, A. G. (2000). Review: tissue engineering for regeneration of articular cartilage. Biomaterials, 21(5), 431-440. doi: http://dx.doi.org/10.1016/S0142-9612(99)00213-6

6. Yang, Y.-Y., Chia, H.-H., \& Chung, T.-S. (2000). Effect of preparation temperature on the characteristics and release profiles of PLGA microspheres containing protein fabricated by double-emulsion solvent extraction/evaporation method. Journal of Controlled Release, 69(1), 8196. doi: http://dx.doi.org/10.1016/S0168-3659(00)00291-1 